\title{
XbotML: A Markup Language for Human Computer Interaction via Chatterbots
}

\author{
André M.M. Neves and Flávia A. Barros \\ Centro de Informática \& VIRTUS - Laboratório de Hipermídia, Universidade Federal de \\ Pernambuco, Caixa Postal 7851 CEP 50732-970 - Recife (PE) - Brazil \\ \{ammn, fab\}@cin.ufpe.br
}

\begin{abstract}
Since 1995, we have been watching the emergence of a new paradigm for the construction of chatterbots based on markup languages. The most prominent of these languages is AIML. Despite its success, XML chatterbots have drawbacks in what concerns the level of fluency in dialogues. We present here XbotML, a new language for the construction of chatterbots based on principles of the Conversational Analysis theory. Following this theory, XbotML structures dialogues between user and chatterbot in adjacency pairs, each pair bearing one associated intention. As proof of concept, we constructed a chatterbot that has reached fluency level around $75 \%$ in dialogues with people. This is an original work that contributes to raise the fluency level of XML chatterbots by: providing a linguistically grounded model for chatterbots' markup languages bases; providing a modular model for these bases; and allowing the extension of existing bases to different domains and applications.
\end{abstract}

\section{Introduction}

The dissemination of the Web stimulated the revival and faster growing of systems that communicate with users in natural language. These systems, currently known as chatterbots [1], have being used in the Internet for the most varied tasks (e.g., to take part on chat rooms and RPG, to sell products, to represent companies, to give technical support, to answer FAQs, to accompany students in distance-learning environments, among others). In this scenario, the aim of chatterbots is to facilitate human computer interaction, since the Web counts on millions of users with different computer literacy levels.

In our research work, we identified three generations of chatterbots (section 2). The first generation was strongly based on pattern-matching techniques, whereas the second generation used Artificial Intelligence techniques. Since 1995, we have been watching the emergence of a third generation of chatterbots, based on the use of markup languages [2]. AIML (Artificial Intelligence Markup Language) [3], the first of these languages, was used in the construction of $\mathrm{ALICE}^{1}$, the owner of two recent Loebner prizes ${ }^{2}$. Despite its success, the robots developed using AIML still present

\footnotetext{
${ }^{1}$ http://www.alicebot.org

${ }^{2}$ http://www.loebner.net/Prizef/loebner-prize.html
} 
problems with fluency in dialogues with humans (reaching levels up to 65\%), which, according to Rooijmans [4], is a central point in the chatterbots development.

Our research work verified two main factors that may cause loss of fluency in this kind of dialogues: (1) the chatterbot does not have domain information about the theme chosen by the user; and (2) the chatterbot is not able to recognize the user sentences' intentions in the dialogue. Clearly, a gap exists in the available markup languages, which should be tackled in order to improve the performance of these robots.

We present here XbotML, a new language for the construction of chatterbots based on principles of the Conversational Analysis theory [5]. Following this theory, XbotML structures dialogues between user and chatterbot in adjacency pairs (stimulus-and-response pairs), each pair bearing one associated intention. For that, XbotML organizes chatterbots' knowledge bases into three different XML patterns (section 3.3). We focused our work on the solution of the second factor mentioned above as contributing to the loss of fluency in dialogues. We believe that the first factor (lack of domain information) is easier to treat, since it can be solved simply by the creation of large bases of pairs (such as in ALICE, which counts on a base of 42.000 categories). As proof of concept, we constructed a chatterbot which has reached fluency level around $75 \%$ in dialogues with people of different ages and interests.

This is an original work that contributes to raise the fluency level of XML chatterbots by: (1) providing a linguistically grounded model for chatterbots' markup languages bases; (2) providing a modular model for these bases; and (3) allowing the extension of existing bases to different domains and applications. This way, our research work aims at contributing to the emergence of a new approach in the construction of chatterbots, raising the markup languages' level of abstraction in order to reach dialogues' fluency rates around $90 \%$.

In section 2 we give a brief description of chatterbots state-of-the-art. In section 3 we present XbotML in detail, and in section 4 we show the experiments results. Section 5 brings conclusions and future work.

\section{Chatterbots in the Cyberspace}

The idea of communicating with machines in natural language dates from 1950, when Turing proposed what was later known as the Imitation Game [6]. Since then, we can identify several attempts of creating such system, from Eliza [7], a landmark in the area, to ALICE, the owner of two recent Loebner Prizes.

After a careful analysis of the area, we identified three main "generations" of chatterbots that differ in what concerns the technological paradigm within which the system was designed and implemented. The first generation can be characterized by the use of pattern-matching techniques of question-answer pairs and grammatical rules. Eliza is, for sure, the most successful system under this paradigm. Some other examples are PC Therapist [8] and FRED [9].

The second generation deploys Artificial Intelligence techniques, such as production rules and neural networks, in the construction of chatterbots. Julia [10], 
which is based on production rules, is probably its most prominent representative. Although this generation used more sophisticated techniques, the obtained results (regarding dialogue fluency) were not superior to the levels obtained in the previous generation. This fact becomes more evident when we observe the results of the Loebner prizes.

The third generation is based on the use of markup languages for the construction of chatterbots' knowledge bases. The proponent of this new paradigm is Dr. Richard Wallace [3], who developed AIML (Artificial Intelligence Markup Language). An AIML knowledge base consists of "categories" of question-answers pairs (see an example of AIML category in figure 1). Due to its relatively simple syntax, AIML is contributing to the popularization of chatterbots in websites ${ }^{3}$.

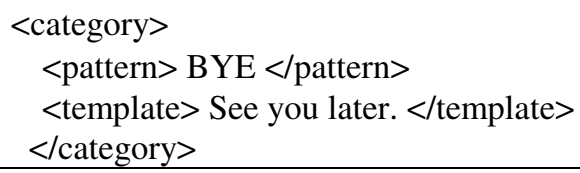

Fig. 1. Example of AIML category

AIML was used in the construction of ALICE (Artificial Linguistic Internet Computer Entity), the owner of the Loebner Prize in 2000 and 2001. Currently, ALICE counts on a base of approximately 42.000 AIML categories. Its interpreter deploys a technique very similar to the traditional pattern matching used in Eliza (question-answer pairs). Despite its good results in the Loebner Prize, ALICE showed some drawbacks in a thorough analysis undergone during the development of our research. Even with its huge categories base, ALICE reached fluency level no higher than $65 \%$. Besides that, its base is flat and therefore very difficult to read and update.

The following section presents XbotML, which tries to overcome these drawbacks, aiming to reach fluency levels of $90 \%$, as well as to improve the readability and extensibility of chatterbots bases.

\section{The XbotML Framework}

In this section, we present XbotML, a new framework for the construction of chatterbots based on principles of the Conversational Analysis theory [5]. XbotML structures dialogues in adjacency pairs, each pair bearing one associated intention. Our main goal is to improve the fluency level in dialogue sessions between users and chatterbots.

This work was initially motivated by the aim of building a chatterbot for the Portuguese language to be used in a distance-learning environment running at VIRTUS (UFPE's Laboratory of Hypermedia) ${ }^{4}$. This research was developed in the context of a larger project, FIACI [11][12], sponsored by Protem-CC/CNPq Brazilian Research Council.

\footnotetext{
${ }^{3}$ See ALICE's Website for details - http://www.alicebot.org

${ }^{4}$ http://www.virtus.ufpe.br
} 
In what follows, we present an overview of our research work (section 3.1), the linguistic concepts underlying this proposal (section 3.2), as well a description of XbotML language (section 3.3). Finally, we show the architecture of the developed processor for XbotML (section 3.4). The practical benefits of this work are illustrated in section 4 , where we present experiments results.

\subsection{Research Development}

The primary aim of our research work is to provide a framework for the construction of chatterbots capable of leading dialogues with high fluency levels (around 90\%). We opted to work within the third generation of chatterbots, which is centered around the use of markup languages for the construction of chatterbots' knowledge bases, due to the clear advantages offered by this paradigm: extensibility, modularity, adequacy to Web applications, among others.

In the quest for a solution to fluency problems in dialogues between chatterbots and users, our research investigated three different approaches to build these systems.

Starting from the state-of-the-art AIML solution, we initially used the AIML processor available for download in ALICE's Website to build a chatterbot for the Portuguese language. As in the original AIML language, the chatterbot's base was formed by a list of AIML categories [11][12]. The implemented chatterbot showed a very low fluency level in dialogues (around 25\%), what motivated us to search for a more appropriate solution (see section 4.1 for details).

A thorough analysis of this initial work revealed that, as in natural conversations, dialogues could be seen as structured according to participants' intentions. We then envisioned the Speech Acts theory [13] as a possibility to structure AIML categories into classes according to their intentions. We extended the syntax of the original AIML language and its processor to cope with classes of intentions, and organized our original categories into 14 classes. The extended language was named as AIMLPlus [14]. The new chatterbot implemented based on these ideas presented much better results - around 50\% in dialogues' fluency level (see section 4.2). After several attempts to improve this rate, a serious drawback was revealed by a deep qualitative analysis: Speech Acts account solely for the intention of the speaker, not considering the hearer's response in one go. This finding put forth the need to review our choices.

Building on that, our next step was to search for a linguistic theory which could account for intentions covering dialogues' basic units, the stimulus-and-response pairs [15], also known as adjacency pairs. This sociolinguistic concept has already been deployed in the field of agents communication (in particular, to model conversational agents) [16]. A careful bibliographic investigation naturally led us to adopt the Conversational Analysis Theory [5] (section 3.2) as the conceptual model upon which to build our chatterbot systems. A new markup language, XbotML (section 3.3), was devised and a new processor was developed (section 3.4) to account for the particularities of this new approach. The previous knowledge base was completely revised according to this new paradigm, resulting on a chatterbot that has reached fluency level around $75 \%$ in dialogues with people of different ages and interests (section 4.3). The obtained fluency rate so far is considered very satisfactory, since not even in dialogues between two humans the fluency always reaches $100 \%$. 


\subsection{Conversational Analysis Theory}

This section briefly presents the Conversational Analysis theory [5], used as the basis for a consistent structuring of chatterbots' bases. Marcuschi [5] proposed that the analysis of conversations (dialogues) should be empiric, starting from single occurrences, aiming to reach general rules that account for different situations. Besides, he treats dialogues in two levels: local and global.

In what concerns the dialogues' local organization, Marcuschi highlights two essential concepts: (1) turn, which represents each contribution of the dialogue's participants while talking; and (2) adjacency pair, a term used "to refer to a single stimulus-plus-response sequence by the participants" [17] in the dialogue. In this approach, speaker and hearer exchange roles as the dialogue unfolds. As such, each participant will play the role of speaker in one turn of the adjacency pair. Each adjacency pair has an intention associated to it, such as invitation-acceptance/refuse, question-answer, etc. The pair's intention may be identified via stereotyped terms (words or expressions) named markers (e.g., what, who, how much). In short, adjacency pairs are sequences of two turns which co-occur and are complementary in an ordered way. The notion of fluency here is guided by the intention associated to the initial contribution.

When analyzing Marcuschi's proposed model, we noticed that the identification of the initial contribution's intention is essential for selection of an adequate response, which could minor fluency problems found in dialogues between humans and chatterbots. However, it was still necessary to identify the adjacency pairs that occur in dialogues between a user and a chatterbot, since they differ from a natural conversation between two humans (see section 4.3 for details).

The dialogues' global organization is view by Marcuschi as a structure which may be divided into several parts. Among them, he highlights three parts that are most usually found in ordinary conversations: (1) the opening session, where the participants in the dialogue introduce themselves and/or greet each other; (2) the development, which focuses on one or more topics; and (3) the closing session, where the participants say goodbye to each other.

Concerning our work, the undergone experiments revealed that dialogues with chatterbot are more similar to conversation over the telephone than to face-to-face dialogues. In the opening session, the user identifies himself/herself to the chatterbot (such as in dialogues over the phone). In the development session, it is also the user who defines the first dialogue's topic, and in the closing session, the user says goodbye first, like in telephone calls (where the one who called usually finishes off the dialogue) [5].

\subsection{XbotML Structure}

We present here the structure of XbotML, a chatterbots markup language based on principles of the Conversational Analysis theory. We centered the language's design around the two major concepts of this theory: turn and conversational pair. 
XbotML consists of four different XML patterns ${ }^{5}$ : (1) the input pattern, consisting of classes of markers which guide the identification of the user's sentence intention, the first contribution of the adjacency pair; (2) the strategy pattern, consisting of classes of if-then rules used to determine the intention of the chatterbot's response, according to the user's sentence intention and to the log records; (3) the output pattern, with classes of chatterbot's response templates, the second contribution in the adjacency pair; and (4) the log pattern, which registers every adjacency pair in the dialogue together with its input and output associated intentions. In contrast to AIML, XbotML structure is not guided by categories, but rather by classes of intentions which aggregate markers in the input pattern, rules in the strategy pattern and response templates in the output pattern.

To illustrate our proposal, we present below examples of XbotML elements for each of the patterns seen above. Initially, figure 2 presents a class in the input pattern that has as intention "say goodbye to the chatterbot". If the user types the sentence "Good bye, I'm too tired", for instance, the system will match this input to the marker "good bye", and the intention of this class will be selected and associated to the input sentence. Similarly, if the user types the sentence "Bye, it's late", the same class will be selected by the system. It illustrates how XbotML deals with several different (but equivalent) input sentences, always selecting the same input class. Clearly, the user's intention in both cases was the same: say goodbye to the chatterbot. The algorithm deployed in the intention's selection process is presented in section 3.4.

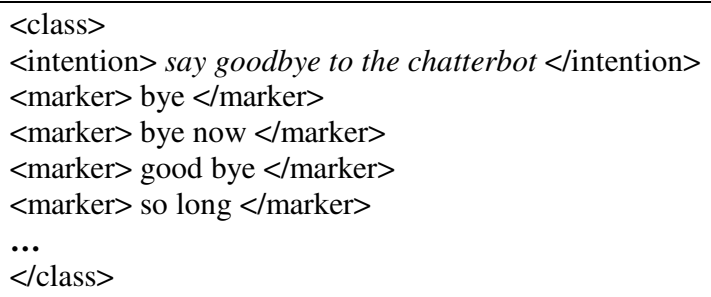

Fig. 2. Example of an XbotML class in the input pattern

The corresponding class in the strategy pattern to the above example is shown in figure 3 . The use of the strategy rules is explained in section 3.4 below.

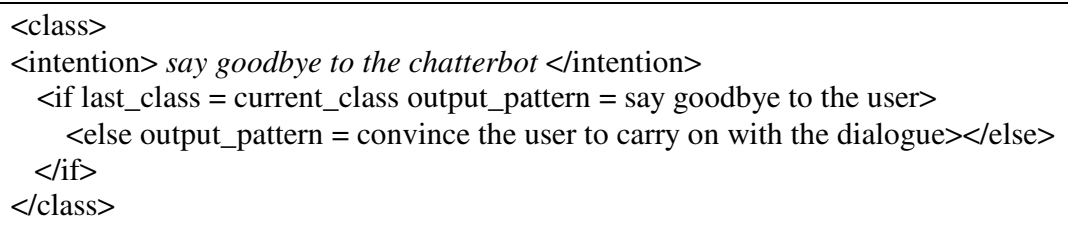

Fig. 3. Example of an XbotML class in the strategy pattern

The corresponding classes in the output pattern to the above example are shown in figure 4. As seen, these classes contain output templates that may have slots to be filled in (in the case below, the username, indicated by the </username $>$ element). 
$<$ class $>$

$<$ intention $>$ say goodbye to the user </intention>

$<$ response $>$ good bye $<$ /username $><$ /response $>$

...

$</$ class $>$

Fig. 4. Example of an XbotML class in the output pattern

\subsection{XbotML Processor}

We present here the general architecture of chatterbots based on XbotML, as well as some implementation details. The XbotML processor counts on the four patterns presented in the above section plus one dictionary of common words abbreviations, and six processing modules, each one with a different functionality.

The Botmaster Interface is used by the chatterbot's manager to create and update the Input, Strategy and Output blocks, as well as to consult the log records. This module is not accessible to end users.

The User Interface receives the user's input and presents the chatterbot's response, this way simulating a chat room. It keeps the full dialogue on the screen, so that the user can always read past contributions. This module is implemented in HTML and can be accessed via any available browser.

The Input Pre-processor translates the user's sentence into a format compatible with the XbotML input markers. Every token in the input string is verified with the help of the system's dictionary, and the recognized abbreviations are replaced by the corresponding full word (for example, "vc" is replaced by "voce" - "you" in Portuguese). Besides, the existing accents are suppressed (e.g., "você" is replaced by "voce") and upper case letters are replaced by lower case. The aim here is to facilitate the matching process.

This internal representation is passed onto the Intentions Classifier module, which is responsible for identifying the input sentence's intention from among the ones available in the Input block. The structure of XbotML patterns is fixed (section 3.3), however, the content of the Input, Strategy, and Output blocks may vary from one chatterbot to another. The decision about what intentions will be included in each block is at the Botmaster's hands.

The identification of the input sentence's intention is based on a pattern matching algorithm specially built for the XbotML processor. This algorithm has three major steps:

(1) first, the algorithm selects all markers in the Input block which perfectly match or include the input sentence (e.g., for the input sentence "bye" the algorithm selects "bye" and "bye now" markers in the example of figure 2); (2) following, the algorithm selects all markers in the Input block included in the input sentence (e.g., for the input sentence "good bye, I'm too tired", the algorithm selects "bye" and "good bye" markers in the example of figure 2); (3) finally, it computes the words in common between every selected marker and the input sentence, and the marker with higher number of words in common to the input sentence is selected (only if the matching rate is above a limit defined by the Botmaster). 
When no match is obtained, this module associates the intention "not recognized" to the input sentence.

Our strategy differs from the one deployed by the AIML interpreter, which always selects the longer category pattern that includes the input sentence. We point out two problems here: (1) the longer category pattern may not always be the most precise match; and, (2) in case no available category includes the input sentence, there will be no possible partial match with a pattern included in the input sentence (which might very well represent its intention).

The Decision Making module receives the input sentence's internal representation together with its associated intention, and determines the class of the response to be built by the Response Composer module. This process is guided by the rules available in the Strategies block (see an example of the system in use below).

The Response Composer receives the input sentence's internal representation and the response's class, and builds the most appropriate reply based on that class' templates available in the Output block. Finally, this module returns the chatterbot's output to the user via the User Interface.

The Log records are created with information provided by the Input Pre-processor (user ID and name, date and input sentence), by the Intentions Classifier module (input class) and by the Response Composer (chatterbot's response and associated class).

The running version of this processor is implemented in $\mathrm{PERL}^{6}$, due to the language offered facilities to deal with text matching and Internet applications.

\section{Experiments Results}

This section describes three major experiments undergone within our work. We implemented three chatterbots for Portuguese language, each one corresponding to one of the approaches described in section 3.1 (Research Development). These experiments were central for the evolution of our research, since they pointed out the drawbacks of each adopted solution.

In what follows, we present details of each implemented chatterbot, together with experiments results. The current version, based on the XbotML, is a standalone application running at VIRTUS Laboratory of Hypermedia ${ }^{7}$. It is worth to point that the dialogues' fluency level of our chatterbots improved from $25 \%$ (in the first version) to $75 \%$ (in the current version).

\subsection{Experiment 01 - An AIML Chatterbot for Portuguese}

The first version of our chatterbot, released in June 2001, bore the same structuring as any other chatterbot in AIML. Its base consisted of a flat list of 2.000 AIML categories for the Portuguese language. Some of these categories were simple translations of standard AIML categories from English to Portuguese, and some were

\footnotetext{
${ }^{6} \mathrm{http}: / / \mathrm{www} \cdot$ perl.com

${ }^{7} \mathrm{http}: / /$ www.virtus.ufpe.br/pixelbot
} 
based on log files of dialogues among users of VIRTUS Website chat rooms ${ }^{8}$. The processor used in this experiment was adapted from the available AIML free Java code to cope with particularities of the Portuguese language (e.g., the use of possessive pronouns and prepositions in verb phrases).

In order to access the system's performance, dialogues between the chatterbot and 143 individual users were analyzed during 30 consecutive days. Regarding quantitative results, we verified that our chatterbot was able to respond with fluency only $25 \%$ of the users' contributions, and that approximately $45 \%$ of the contributions were classified as "ignored", in which case the chatterbot returned generic responses such as "would you like to change subject?".

On the other hand, a qualitative analysis of the log files of this experiment revealed that the users' contributions could be grouped into classes, according to their intentions. This analysis identified 14 different users' intentions when communicating with our chatterbot (see section 4.2). This finding was the main motivation for the creation of AIML-Plus (section 3.1).

\subsection{Experiment 02 - An AIML-Plus Chatterbot for Portuguese}

As said above, a thorough analysis of the log files obtained during experiment 01 revealed that the users' contributions were not random, but rather they could be grouped according to their intentions. We identified 14 different intentions among the users' contributions, as following: to start a dialogue with the robot, to thank the robot, to compliment the robot, to insult the robot, to ask how the robot is feeling, to claim something, to ask a general question to the robot, to ask the robot to define a specific issue, to ask who is a specific person, to affirm that he/she understands the robot, to affirm that he/she is charmed by the robot, to ask personal questions to the chatterbot (e.g., where it lives, where it works, where it was born, who created it etc), to ask how it is codified, and to say good-bye to the robot.

Based on this analysis, and inspired by the Speech Acts theory [13], we grouped the original AIML categories into classes according to their intentions. The initial 2.000 categories were then classified into the 14 intentions identified during our analysis. In order to implement this new approach, it was necessary to extend the original AIML language such that it could cope with some structuring on its categories base. This extension was named AIML-Plus [14]. The main modifications to the original AIML format were: (1) the creation of a new element, <class>, to aggregate categories with the same intention; and (2) the inclusion of different, however equivalent, input patterns in each category, to improve fluency. The previous processor had to be modified to deal with the implemented extensions.

The AIML-Plus version was released in September 2001 in VIRTUS Website. This experiment ran during 12 months, having in average 200 users a day. The recorded dialogues formed a corpus for analysis of approximately 3.000 sentences daily.

Qualitative and quantitative analyses of this data revealed a fluency level around $50 \%$ in dialogues between chatterbot and users. This chatterbot presented much better

${ }^{8}$ http://www.virtusclass.org 
results than its predecessor. However, despite several efforts, this rate could not be improved. As seen in section 3.1, we verified that a new linguistic model was necessary to overcome this problem.

\subsection{Experiment 03 - An XbotML Chatterbot for Portuguese}

Following our new research directions, another chatterbot was created based on XbotML. The chatterbot's base is now organized within three different blocks (Input, Strategy and Output), which substitute the previous class-categories base. The processor had to be rewritten, since the previous base structure had to be radically modified (section 3.4). The available log files were analyzed and 45 adjacency pair classes of intentions were identified as the most usual ones in dialogues between chatterbots and users. This version is available at VIRTUS Website. The XbotML chatterbot is still under test, and has reached fluency level superior to $75 \%$ so far.

\section{Conclusion and Future Work}

We presented here XbotML, a new language for the construction of chatterbots. This is an original work whose main aim is to raise the fluency of XML chatterbots to levels around $90 \%$. For that, we adopted a linguistically grounded model for XbotML bases (based on principles of the Conversational Analysis theory), favoring the modularity and extensibility of these bases. The results obtained with our experiments clearly show that we are in the right track. Currently, the available XbotML base for Portuguese is being augmented, and we expect to reach higher levels of fluency (around 85\%) within the next six month.

As future work, we can point out several simultaneous extensions to XbotML. At the moment, we are working on four different subprojects: (1) the development of a tool for the semi-automatic learning of new markers (to augment the current XbotML input block); (2) the construction of a chatterbot capable of communicating in several languages at the same time (e.g., English and Portuguese) to be used as an interpreter in chat rooms with speakers of different languages; (3) the incorporation of new XbotML elements which provide for chatterbots with personality and mood; and (4) the construction of a chatterbot that serves as a natural language interface to a system which recommends films to its users based on profiles. In this application, the chatterbot is also responsible for automatically acquiring the user's profile from the undergone dialogues.

\section{References}

1. Laven, S.: Online at http://www.simonlaven.com

2. W3schools: Online at http://www.w3schools.com/xml

3. Wallace, R. S.: Don't read me - A.L.I.C.E. and AIML documentation. Online at http://www.alicebot.com/dont.html 
4. Rooijmans, B:. Believable Agents. Master's thesis at Faculty of Science, University of Amsterdam, 2000

5. Marcuschi, L.A.: Análise da Conversação. Editora Ática, São Paulo, 1986

6. Turing, A. M.: Computing machinery and intelligence. Mind, vol. 59 n. 236:433-460.

7. Weizenbaum, J. ELIZA: A computer program for the study of Natural Language Communication between man and machine. In Communications of ACM, vol. 9, n.1, 1966.

8. Weintraub, J.: History of the PC Therapist Online at http://www.loebner.net/Prizef/weintraub-bio.html

9. Garner, G.R.: Generation5, 2000. Online at http://www.generation5.org/garner.shtml

10. Foner, L. N.: What's an agent, anyway? A sociological case study. Online at http://foner.www.media.mit.edu/people/foner/Julia/Julia.html.

11. Barros, F.A., Neves, A.M.M., Costa, F.P.D. \& Cavalcante, P.S.: FIACI: A Methodology for the Constructions of Intelligent Tools for Cooperative Learning in the Internet. In Prugner, N, Costa, C.D. \& Lima, P.M. (eds.) Proceedings of ProTem-CC Projects Evaluation Workshop - Informatics in Education. Pp 161-175. October 1-3, Rio de Janeiro (RJ), Brasil. 2001.

12. Neves, A.M.M, Paraguaçu, F., Barros, F., Cavalcante, P.S. \& Barros, S.G. Projeto FIACI: Concepção de Ferramentas Inteligentes para Aprendizagem Cooperativa na Internet. In Anais do XII Simpósio Brasileiro de Informática na Educação. Vitória (ES) Brasil. Nov/2001.

13. Searle J. R.: Expression and Meaning. Cambridge University Press. 1979

14. Neves, A. M. M., Diniz, I., Barros, F. A.: Natural Language Communication via AIML Plus Chatterbots In: V Symposium on Human Factors in Computer Systems (IHC 2002), 2002, Fortaleza - CE. Proc. of the IHC 2002 - V Symposium on Human Factors in Computer Systems 2002. , 2002. p.387

15. Schegolof, Emanuel E.: Sequencing in Conversational Openings, New York 1972

16. Zacharski, R.: Conversational Agents for Language Learning. in Innovative Applications of Artificial Intelligence Conference 2002, New Mexico.

17. Crystal, D. A.: Dictionary of Linguistics and Phonetics. 3rd edition. Basil Blackwell Ltda. 1991. 